\title{
Internet of Things (IOT): Research Challenges and Future Applications
}

\author{
AbdelRahman H. Hussein \\ Department of Networks and Information Security \\ Faculty of Information Technology / Al-Ahliyya Amman University
}

\begin{abstract}
With the Internet of Things (IoT) gradually evolving as the subsequent phase of the evolution of the Internet, it becomes crucial to recognize the various potential domains for application of IoT, and the research challenges that are associated with these applications. Ranging from smart cities, to health care, smart agriculture, logistics and retail, to even smart living and smart environments IoT is expected to infiltrate into virtually all aspects of daily life. Even though the current IoT enabling technologies have greatly improved in the recent years, there are still numerous problems that require attention. Since the IoT concept ensues from heterogeneous technologies, many research challenges are bound to arise. The fact that IoT is so expansive and affects practically all areas of our lives, makes it a significant research topic for studies in various related fields such as information technology and computer science. Thus, IoT is paving the way for new dimensions of research to be carried out. This paper presents the recent development of IoT technologies and discusses future applications and research challenges.
\end{abstract}

Keywords-Internet of Things; IoT applications; IoT challenges; future technologies; smart cities; smart environment; smart agriculture; smart living

\section{INTRODUCTION}

The Internet can be described as the communication network that connects individuals to information while The Internet of Things (IoT) is an interconnected system of distinctively address able physical items with various degrees of processing, sensing, and actuation capabilities that share the capability to interoperate and communicate through the Internet as their joint platform [1]. Thus, the main objective of the Internet of Things is to make it possible for objects to be connected with other objects, individuals, at any time or anywhere using any network, path or service. The Internet of Things (IoT) is gradually being regarded as the subsequent phase in the Internet evolution. IoT will make it possible for ordinary devices to be linked to the internet in order to achieve countless disparate goals. Currently, an estimated number of only $0.6 \%$ of devices that can be part of IoT has been connected so far [2]. However, by the year 2020, it is likely that over 50 billion devices will have an internet connection.

As the internet continues to evolve, it has become more than a simple network of computers, but rather a network of various devices, while IoT serves as a network of various "connected" devices a network of networks [3], as shown in Fig. 1. Nowadays, devices like smartphones, vehicles, industrial systems, cameras, toys, buildings, home appliances, industrial systems and countless others can all share information over the Internet. Regardless of their sizes and functions, these devices can accomplish smart reorganizations, tracing, positioning, control, real-time monitoring and process control. In the past years, there has been an important propagation of Internet capable devices. Even though its most significant commercial effect has been observed in the consumer electronics field; i.e. particularly the revolution of smartphones and the interest in wearable devices (watches, headsets, etc.), connecting people has become merely a fragment of a bigger movement towards the association of the digital and physical worlds.

With all this in mind, the Internet of Things (IoT) is expected to continue expanding its reach as pertains the number of devices and functions, which it can run. This is evident from the ambiguity in the expression of "Things" which makes it difficult to outline the ever-growing limits of the IoT [4]. While commercial success continues to materialize, the IoT constantly offers a virtually limitless supply of opportunities, not just in businesses but also in research. Accordingly, the understudy addresses the various potential areas for application of IoT domains and the research challenges that are associated with these applications.

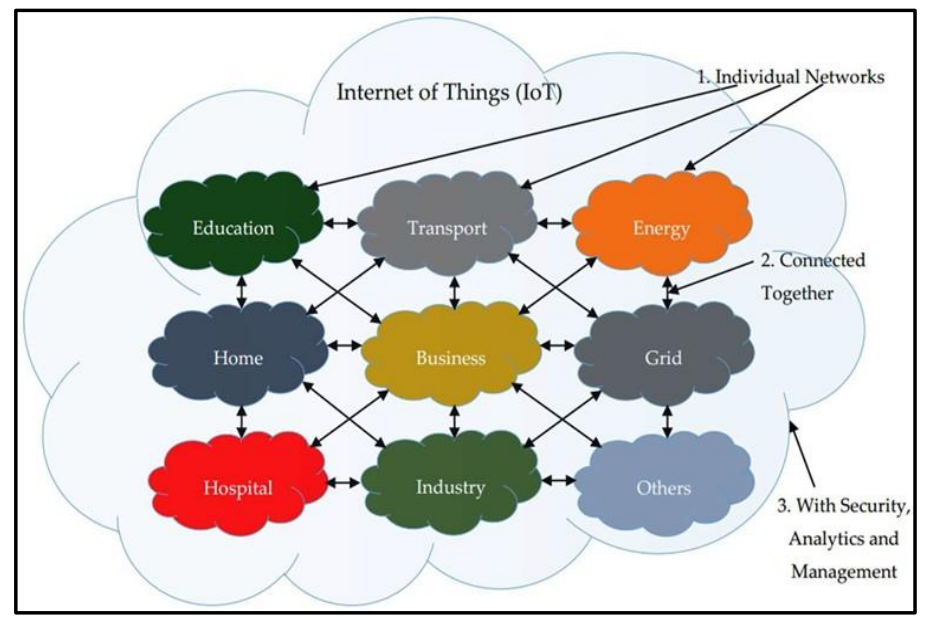

Fig. 1. IoT can be viewed as a Network of Networks [3].

\section{Potential Application Domains of IoT}

Potential applications of the internet of Things are not only numerous but also quite diverse as they permeate into virtually all aspects of daily life of individuals, institutions, and society. According to [5], the applications of IoT cover broad areas including manufacturing or the industrial sector, health sector, agriculture, smart cities, security and emergencies among many others. 


\section{A. Smart Cities}

According to [6], the IoT plays a crucial role in improving the smartness of cities and enhancing general infrastructure. Some of IoT application areas in creating smart cities include; intelligent transportation systems [7], smart building, traffic congestion [7, 8] waste management [9], smart lighting, smart parking, and urban maps. This may include different functionalities such as; monitoring available parking spaces within the city, monitoring vibrations as well as material conditions of bridges and buildings, putting in place sound monitoring devices in sensitive parts of cities, as well as monitoring the levels of pedestrians and vehicles. Artificial Intelligence (AI) enabled IoT can be utilized to monitor, control and reduce traffic congestions in Smart Cities [6]. Moreover, IoT allows installation of intelligent and weather adaptive street lighting and detection waste and waste containers by keeping tabs of trash collection schedules. Intelligent highways can provide warning messages and important information, such as access to diversions depending on the climatic conditions or unexpected occurrences like traffic jams and accidents.

Application of IoT to achieve smart cities would require using radio frequency identification and sensors. Some of the already developed applications in this area are the Aware home and the Smart Santander functionalities. In the United States, some major cities like Boston have plans on how to implement the Internet of Things in most of their systems ranging from their parking meters, streetlights, sprinkler systems, and sewage grates are all scheduled to be interlinked and connected to the internet. Such applications will offer significant break throughs in terms of saving money and energy.

\section{B. Healthcare}

Most healthcare systems in many countries are inefficient, slow and inevitably prone to error. This can easily be changed since the healthcare sector relies on numerous activities and devices that can be automated and enhanced through technology. Additional technology that can facilitate various operations like report sharing to multiple individuals and locations, record keeping and dispensing medications would go a long way in changing the healthcare sector [10].

A lot of benefits that IoT application offers in the healthcare sector is most categorized into tracking of patients, staff, and objects, identifying, as well as authenticating, individuals, and the automatic gathering of data and sensing. Hospital workflow can be significantly improved once patients flow is tracked. Additionally, authentication and identification reduce incidents that may be harmful to patients, record maintenance and fewer cases of mismatching infants. In addition, automatic data collection and transmission is vital in process automation, reduction of form processing timelines, automated procedure auditing as well as medical inventory management. Sensor devices allow functions centered on patients, particularly, in diagnosing conditions and availing real-time information about patients' health indicators [6].

Application domains in this sector include; being able to monitor a patient's compliance with prescriptions, telemedicine solutions, and alerts for patients' well-being. Thereby, sensors can be applied to outpatient and inpatient patients, dental Bluetooth devices and toothbrushes that can give information after they are used and patient's surveillance. Other elements of IoT in this capacity include; RFID, Bluetooth, and Wi-Fi among others. These will greatly enhance measurement and monitoring techniques of critical functions like blood pressure, temperature, heart rate, blood glucose, cholesterol levels, and many others.

The applications of Internet of Things (IoT) and Internet of Everything (IoE) are further being extended through the materialization of the Internet of Nano-things (IoNT) [3]. The notion of IoNT, as the name implies, is being engineered by integrating Nano-sensors in diverse objects (things) using Nano networks. Medical application, as shown in Fig. 2, is one of the major focuses of IoNT implementations. Application of IoNT in human body, for treatment purposes, facilitates access to data from in situ parts of the body which were hitherto in accessible to sense from or by using those medical instruments incorporated with bulky sensor size. Thus, IoNT will enable new medical data to be collected, leading to new discoveries and better diagnostics.

\section{Smart Agriculture and Water Management}

According to [11], the IoT has the capacity to strengthen and enhance the agriculture sector through examining soil moisture and in the case of vineyards, monitoring the trunk diameter. IoT would allow to control and preserve the quantity of vitamins found in agricultural products, and regulate microclimate conditions in order to make the most of the production of vegetables and fruits and their quality. Furthermore, studying weather conditions allows forecasting of ice information, drought, wind changes, rain or snow, thus controlling temperature and humidity levels to prevent fungus as well as other microbial contaminants.

When it comes to cattle, IoT can assist in identifying animals that graze in open locations, detecting detrimental gases from animal excrements in farms, as well as controlling growth conditions in offspring to enhance chances of health and survival and so on. Moreover, through IoT application in agriculture, a lot of wastage and spoilage can be avoided through proper monitoring techniques and management of the entire agriculture field. It also leads to better electricity and water control.

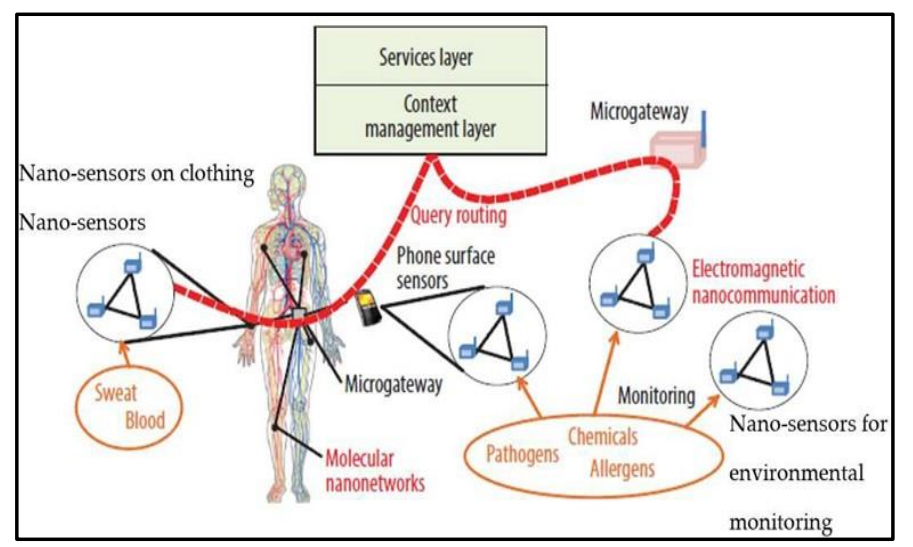

Fig. 2. The Internet of Nano-Things [3]. 
As [11] explain, in water management, the role of IoT includes studying water suitability in seas and rivers for both drinking and agriculture use, detecting pressure variations in pipes, and liquid presence outside tanks as well as monitoring levels of water variation in dams, rivers and reservoirs. These IoT applications utilize Wireless sensor networks. Examples of existing IoT applications in this domain include; SiSviA, GBROOS, and SEMAT.

\section{Retail and Logistics}

Executing the IoT in Supply Chain or retail Management has many benefits. Some include; observing storage conditions throughout the supply chain, product tracking to enable trace ability purposes, payment processing depending on the location or activity period in public transport, theme parks, gyms, and others. Inside the retail premises, IoT can be applied to various applications such as direction in the shop based on a preselected list, fast payment processes like automatically checking out with the aid of biometrics, detecting potential allergen products and controlling the rotation of products on shelves and warehouses in order to automate restocking procedures [12].

The IoT elements mostly used in this setting include; wireless sensor networks and radio frequency identification. In retail, there is a current use of SAP (Systems Applications and Products), while in logistics numerous examples include quality consignment conditions, item location, detecting storage incompatibility issues, fleet tracking among others. In the industry domain, IoT helps in detecting levels of gas and leakages within the industry and its environs, keeping track of toxic gases as well as the oxygen levels within the confines of chemical plants to ensure the safety of goods and workers and observing levels of oil, gases and water in cisterns and storage tanks. Application of IoT also assists in maintenance and repair because systems can be put in place to predict equipment malfunctions and at the same automatically schedule periodic maintenance services before there is a failure in the equipment. This can be achieved through the installation of sensors inside equipment or machinery to monitor their functionality and occasionally send reports.

\section{E. Smart Living}

In this domain, IoT can be applied in remote control devices whereby one can remotely switch appliances on and off hence preventing accidents as well as saving energy [1, 3]. Other smart home appliances include refrigerators fitted with LCD (Liquid Crystal Display) screens, enabling one to know what is available inside, what has over stayed and is almost expiring as well as what needs to be restocked. This information can also be linked to a smartphone application enabling one to access it when outside the house and therefore buy what is needed. Furthermore, washing machines can allow one to remotely monitor laundry. In addition, a wide range of kitchen devices can be interfaced through a smartphone, hence making it possible to adjust temperature, like in the case of an oven. Some ovens which have a self-cleaning feature can be easily monitored as well. In terms of safety in the home, IoT can be applied through alarm systems and cameras can be installed to monitor and detect window or door openings hence preventing intruders [3].

\section{F. Smart Environment}

The environment has a vital role within all aspects of life, from people, to animals, birds and also plants, are all affected by an unhealthy environment in one way or another. There have been numerous efforts to create a healthy environment in terms of eliminating pollution and reducing wastage of resources, but the existence of industries, as well as transportations wastes coupled with reckless and harmful human actions are common place elements which consistently damage the environment. Consequently, the environment requires smart and innovative ways to help in monitoring and managing waste, which provide a significant amount of data that forces governments to put in place systems that will protect the environment.

Smart environment strategies integration with IoT technology should be created for sensing, tracking and assessment of objects of the environment that offer potential benefits in achieving a sustainable life and a green world. The IoT technology allows observing and managing of air quality through data collection from remote sensors across cities and providing round the clock geographic coverage to accomplish better ways of managing traffic jams in major cities. Additionally, IoT technology can be applied in measuring pollution levels in water and consequently enlighten decisions on water usage. In waste management, which consists of various types of waste, like chemicals and pollutants being detrimental to the environment and to people, animals, and plants as well, IoT can also be applied. This can be achieved by environmental protection by means of controlling industrial pollution through instantaneous monitoring and management systems combined with supervision in addition to decision making networks. This serves to lessen waste [13].

In weather forecasting, IoT can be used to deliver a significant accuracy and high resolution for monitoring the weather by information sharing and data exchange. Through IoT technology, weather systems can collect information such as barometric pressure, humidity, temperature, light, motion and other information, from vehicles in motion and transmit the information wirelessly to weather stations. The information is attained by installing sensors on the vehicles and even on buildings after which it is stored and analyzed to assist in weather forecasting. Radiation is also a threat to the environment, human and animal health as well as agricultural productivity. IoT sensor networks can control radiation through constant monitoring of its levels, particularly around nuclear plant premises for detecting leakage and propagating deterrence.

\section{RESEARCH CHALlenges}

For all the above potential applications of IoT, there has to be proper feasibility into the different domains to ascertain the success of some applications and their functionality. As with any other form of technology or innovation, IoT has its challenges and implications that must be sorted out to enable mass adoption. Even though the current IoT enabling technologies have greatly improved in the recent years, there are still numerous problems that require attention, hence paving the way for new dimensions of research to be carried out. Since the IoT concept ensues from heterogeneous 
technologies that are used in sensing, collecting, action, processing, inferring, transmitting, notifying, managing, and storing of data, a lot of research challenges are bound to arise. These research challenges that require attention have consequently spanned different research areas [14].

\section{A. Privacy and Security}

Owing to the fact that IoT has become a vital element as regards the future of the internet with its increased usage, it necessitates a need to adequately address security and trust functions. Researchers are aware of the weaknesses which presently exist in many IoT devices. Furthermore, the foundation of IoT is laid on the existing wireless sensor networks (WSN), IoT thus architecturally inherits the same privacy and security issues WSN possesses [3, 15]. Various attacks and weaknesses on IoT systems prove that there is indeed a need for wide ranging security designs which will protect data and systems from end to end. Many attacks generally exploit weaknesses in specific devices thereby gaining access into their systems and consequently making secure devices vulnerable $[16,17]$. This security gap further motivates comprehensive security solutions that consist of research that is efficient in applied cryptography for data and system security, non-cryptographic security techniques as well as frameworks that assist developers to come up with safe systems on devices that are heterogeneous.

There is a need for more research to be conducted on cryptographic security services that have the capability to operate on resource constrained IoT devices. This would enable different skilled users to securely use and deploy IoT systems regardless of the inadequate user interfaces that are available with almost all IoT devices. In addition to the protection and security aspects of the IoT, additional areas like confidentiality in communication, trustworthiness, and authenticity of communication parties, and message integrity, and supplementary safety requirements should also be incorporated. These may include features like being able to prevent communication of various parties. As an example, in business transactions, smart objects must be prevented from facilitating competitors' access to confidential information in the devices and thus using this information maliciously.

\section{B. Processing, Analysis and Management of Data}

The procedure for processing, analysis and data management is tremendously challenging because of the heterogeneous nature of IoT, and the large scale of data collected, particularly in this era of Big Data [18]. Currently, most systems utilize centralized systems in offloading data and carrying out computationally intensive tasks on an international cloud platform. Nevertheless, there is a constant concern about conventional cloud architectures not being effective in terms of transferring the massive volumes of data that are produced and consumed by IoT enabled devices and to be able further support the accompanying computational load and simultaneously meet timing constraints [19]. Most systems are therefore relying on current solutions such as mobile cloud computing and fog computing which are both based on edge processing, to mitigate this challenge.
Another research direction as regards data management is applying Information Centric Networking (ICN) in the IoT. Since these information centric systems offer support in the efficient content retrieval and access to services, they appear to be quite valuable not just in accessing but also transferring as well as managing generated content and its transmission. This solution, however, brings about various challenges such as; how to extend the ICN paradigm competently over the fixed network edge, how to take in IoTs static and mobile devices as well as how to apportion the functionality of ICN on resource constrained devices [19].

Data analysis and its context not only plays a crucial role in the success of IoT, it also poses major challenges. Once data has been collected it has to be used intelligently in order to achieve smart IoT functions. Accordingly, the development of machine learning methods and artificial intelligence algorithms, resultant from neural works, genetic algorithms, evolutionary algorithms, and many other artificial intelligence systems are essential in achieving automated decision making.

\section{Monitoring and Sensing}

Even if technologies concerned with monitoring and sensing have made tremendous progress, they are constantly evolving particularly focusing on the energy efficiency and form aspect. Sensors and tags are normally expected to be active constantly in order to obtain instantaneous data, this aspect makes it essential for energy efficiency especially in lifetime extension. Simultaneously, new advances in nanotechnology/biotechnology and miniaturization have allowed the development of actuators and sensors at the Nanoscale.

\section{M2M (Machine to Machine) Communication and Communication Protocols}

While there are already existing IoT oriented communication protocols like Constrained Application Protocol (CoAP) and Message Queuing Telemetry Transport (MQTT), there is still no standard for an open IoT. Although all objects require connectivity, it is not necessary for every object to be made internet capable since they only need to have a certain capability to place their data on a particular gateway. Additionally, there are a lot of options in terms of suitable wireless technologies such as LoRa, IEEE 802.15.4, and Bluetooth even though it is not clear whether these available wireless technologies have the needed capacity to continue covering the extensive range of IoT connectivity henceforth.

The communication protocols for devices are the driving force in actualizing IoT applications, and they form the main support of data flow between sensors and the physical objects or outer world. While various MAC protocols have been projected for several domains with Frequency Division Multiple Access, Time Division Multiple Access and Carrier Sense Multiple Access (FDMA, TDMA and CSMA) for low traffic efficiency that is collision free, more circuitry in nodes are required respectively. The main objectives of the transport layer include guaranteeing an end-to-end reliability as well as performing end-to-end control of congestion. In this aspect, most protocols are unable to cooperate appropriate end to end reliability [20]. 


\section{E. Blockchain of Things (BCoT): Fusion of Blockchainand Internet of Things}

Similar to IoT, blockchain technologies have also gained tremendous popularity since its introduction in 2018. Even though blockchain was first implemented as an underlying technology of Bitcoin cryptocurrency, it is now being used in multifaceted nonmonetary applications [21]. Miraz argues that both IoT and Blockchain can strengthen each other, in a reciprocal manner, by eliminating their respective inherent architectural limitations [22]. The underlying technology of IoT is WSN. Therefore, analogous to WSN, IoT also suffers from security and privacy issues. On the contrary, the primary reasons for blockchain's implementation trend in nonmonetary applications is due to its inbuilt security, immutability, trust and transparency. These attributes are powered by blockchain's consensus approach and utilization of Distributed Ledger Technologies (DLTs) which require extensive dependency on participating nodes. Therefore, the fusion of these two technologies Blockchain and Internet of Things (IoT) conceives a new notion i.e. the Blockchain of Things (BCoT) where blockchain strengthens IoT by providing extra layer of security while the "things" of IoT can serve as participating nodes for blockchain ecosystems [22]. Thus, blockchain enabled IoT ecosystems will provide enhanced overall security [23] as well as benefit from each other.

\section{F. Interoperability}

Traditionally as regards the internet, interoperability has always been and continues to be a basic fundamental value because the initial prerequisite in Internet connectivity necessitates that "connected" systems have the ability to "speak a similar language" in terms of encodings and protocols. Currently, various industries use a variety of standards in supporting their applications. Due to the large quantities and types of data, as well as heterogeneous devices, using standard interfaces in such diverse entities is very important and even more significant for applications which support cross organizational, in addition to a wide range of system limitations. Therefore, the IoT systems are meant towards being designed to handle even higher degrees of interoperability [24].

\section{CONCLUSION}

The IoT can best be described as a CAS (Complex Adaptive System) that will continue to evolve hence requiring new and innovative forms of software engineering, systems engineering, project management, as well as numerous other disciplines to develop it further and manage it the coming years. The application areas of IoT are quite diverse to enable it to serve different users, who in turn have different needs. The technology serves three categories of users, individuals, the society or communities and institutions. As discussed in the application section of this research paper, the IoT has without a doubt a massive capability to be a tremendously transformative force, which will, and to some extent does already, positively impact millions of lives worldwide. According to [25], this has become even more evident, as different governments around the world have shown an interest in the IoT concept by providing more funding in the field that is meant to facilitate further research. A good example is the Chinese Government.
Countless research groups have been, and continue to be, initiated from different parts of the world, and their main objective is to follow through IoT related researches. As more and more research studies are conducted, new dimensions to the IoT processes, technologies involved and the objects that can be connected, continue to emerge, further paving way for much more application functionalities of IoT. The fact that IoT is so expansive and affects practically all areas of our lives, makes it a significant research topic for studies in various related fields such as information technology and computer science. The paper highlights various potential application domains of the internet of things and the related research challenges.

\section{REFERENCES}

[1] M. H. Miraz, M. Ali, P. S. Excell, and R. Picking, "A Review on Internet of Things (IoT), Internet of Everything (IoE) and Internet of Nano Things (IoNT)", in 2015 Internet Technologies and Applications (ITA), pp. 219-224, Sep. 2015, DOI: 10.1109/ITechA.2015.7317398.

[2] P. J. Ryan and R. B. Watson, "Research Challenges for the Internet of Things: What Role Can OR Play?," Systems, vol. 5, no. 1, pp. 1-34, 2017.

[3] M. Miraz, M. Ali, P. Excell, and R. Picking, "Internet of Nano-Things, Things and Everything: Future Growth Trends", Future Internet, vol. 10, no. 8, p. 68, 2018, DOI: 10.3390/fi10080068.

[4] E. Borgia, D. G. Gomes, B. Lagesse, R. Lea, and D. Puccinelli, "Special issue on" Internet of Things: Research challenges and Solutions".," Computer Communications, vol. 89, no. 90, pp. 1-4, 2016.

[5] K. K. Patel, S. M. Patel, et al., "Internet of things IOT: definition, characteristics, architecture, enabling technologies, application future challenges," International journal of engineering science and computing, vol. 6, no. 5, pp. 6122-6131, 2016.

[6] S. V. Zanjal and G. R. Talmale, "Medicine reminder and monitoring system for secure health using IOT," Procedia Computer Science, vol. 78, pp. 471-476, 2016.

[7] R. Jain, "A Congestion Control System Based on VANET for Small Length Roads", Annals of Emerging Technologies in Computing (AETiC), vol. 2, no. 1, pp. 17-21, 2018, DOI: 10.33166/AETiC.2018.01.003.

[8] S. Soomro, M. H. Miraz, A. Prasanth, M. Abdullah, "Artificial Intelligence Enabled IoT: Traffic Congestion Reduction in Smart Cities," IET 2018 Smart Cities Symposium, pp. 81-86, 2018, DOI: 10.1049/cp.2018.1381.

[9] Mahmud, S. H., Assan, L. and Islam, R. 2018. "Potentials of Internet of Things (IoT) in Malaysian Construction Industry", Annals of Emerging Technologies in Computing (AETiC), Print ISSN: 2516-0281, Online ISSN: 2516-029X, pp. 44-52, Vol. 2, No. 1, International Association of Educators and Researchers (IAER), DOI: 10.33166/AETiC.2018.04.004.

[10] Mano, Y., Faical B. S., Nakamura L., Gomes, P. G. Libralon, R. Meneguete, G. Filho, G. Giancristofaro, G. Pessin, B. Krishnamachari, and Jo Ueyama. 2015. Exploiting IoT technologies for enhancing Health Smart Homes through patient identification and emotion recognition. Computer Communications, 89.90, (178-190). DOI: 10.1016/j.comcom.2016.03.010.

[11] V. Sundareswaran and M. S. null, "Survey on Smart Agriculture Using IoT," International Journal of Innovative Research in Engineering \& Management (IJIREM), vol. 5, no. 2, pp. 62-66, 2018.

[12] P. Tadejko, "Application of Internet of Things in logistics-current challenges,” Ekonomia i Zarz $\left\{a_{\iota}\right\}$ dzanie, vol. 7, no. 4, pp. 54-64, 2015.

[13] S. Rajguru, S. Kinhekar, and S. Pati, "Analysis of internet of things in a smart environment," International Journal of Enhanced Research in Man-agement and Computer Applications,vol. 4, no. 4, pp. 40-43, 2015.

[14] H. U. Rehman, M. Asif, and M. Ahmad, "Future applications and research challenges of IOT," in 2017 International Conference on Informa-tion and Communication Technologies (ICICT), pp. 68-74, Dec 2017. 
[15] Z. Alansari, N. B. Anuar, A. Kamsin, M. R. Belgaum, J. Alshaer, S. Soomro, and M. H. Miraz, "Internet of Things: Infrastructure, Architecture, Security and Privacy", in 2018 International Conference on Com- puting, Electronics Communications Engineering (iCCECE), pp. 150- 155, Aug 2018, DOI: 10.1109/iCCECOME.2018.8658516.

[16] J. A. Chaudhry, K. Saleem, P. S. Haskell-Dowland, and M. H. Miraz, "A Survey of Distributed Certificate Authorities in MANETs," Annals of Emerging Technologies in Computing (AETiC), vol. 2, no. 3, pp. 1118, 2018, DOI: 10.33166/AETiC.2018.03.002.

[17] A. S. A. Daia, R. A. Ramadan, and M. B. Fayek, "Sensor Networks Attacks Classifications and Mitigation", Annals of Emerging Technologies in Computing (AETiC), vol. 2, no. 4, pp. 28-43, 2018, DOI: 10.33166/AETiC.2018.04.003.

[18] Z. Alansari, N. B. Anuar, A. Kamsin, S. Soomro, M. R. Belgaum, M. H. Miraz, and J. Alshaer, "Challenges of Internet of Things and Big Data Integration", in Emerging Technologies in Computing (M. H. Miraz, P. Ex- cell, A. Ware, S. Soomro, and M. Ali, eds.), (Cham), pp. 47-55, Springer International Publishing, 2018, DOI: 10.1007/978-3-31995450-9_4.

[19] J. Cooper and A. James, "Challenges for database management in the internet of things"IETE Technical Review,vol.26,no.5,pp.320-329,2009.

[20] D. B. Ansari, A.-U. Rehman, and R. Ali, "Internet of Things (IoT) Proto- cols: A Brief Exploration of MQTT and CoAP," International Journal of Computer Applications, vol. 179, pp. 9-14, 032018.
[21] M. H. Miraz and M. Ali, "Applications of Blockchain Technology beyond Cryptocurrency", Annals of Emerging Technologies in Computing (AETiC), vol. 2, no. 1, pp. 1-6, 2018, DOI: 10.33166/AETiC.2018.01.001.

[22] Miraz, M.H., "Blockchain of Things (BCoT): The Fusion of Blockchain and IoT Technologies", Advanced Applications of Blockchain Technology, Studies in Big Data 60, 2019, DOI: 10.1007/978-981-138775-3_7, https://doi.org/10.1007/978-981-13-8775-3_7.

[23] Miraz, M. H. and Ali, M., 2018. Blockchain Enabled Enhanced IoT Ecosystem Security, Proceedings of the International Conference on Emerging Technologies in Computing 2018, London Metropolitan University, UK, Part of the Lecture Notes of the Institute for Computer Sciences, Social Informatics and Telecommunications Engineering (LNICST), vol. 200, pp. 38-46, Online ISBN: 978-3-319-95450-9, Print ISBN: 978-3-319-95449-3, Series Print ISSN: 1867-8211, Series Online ISSN: 1867-822X, DOI: 10.1007/978-3-319-95450-9_3, SpringerVerlag, https://link.springer.com/chapter/10.1007/978-3-319-95450-9_3.

[24] A. Mazayev, J. A. Martins, and N. Correia, "Interoperability in IoT Through the Semantic Profiling of Objects," IEEE Access, vol. 6, pp. 19379-19385, 2018.

[25] R. Porkodi and V. Bhuvaneswari, "The Internet of Things (IoT) Applications and Communication Enabling Technology Standards: An Overview," in 2014 International Conference on Intelligent Computing Applications, pp. 324-329, March 2014. 